
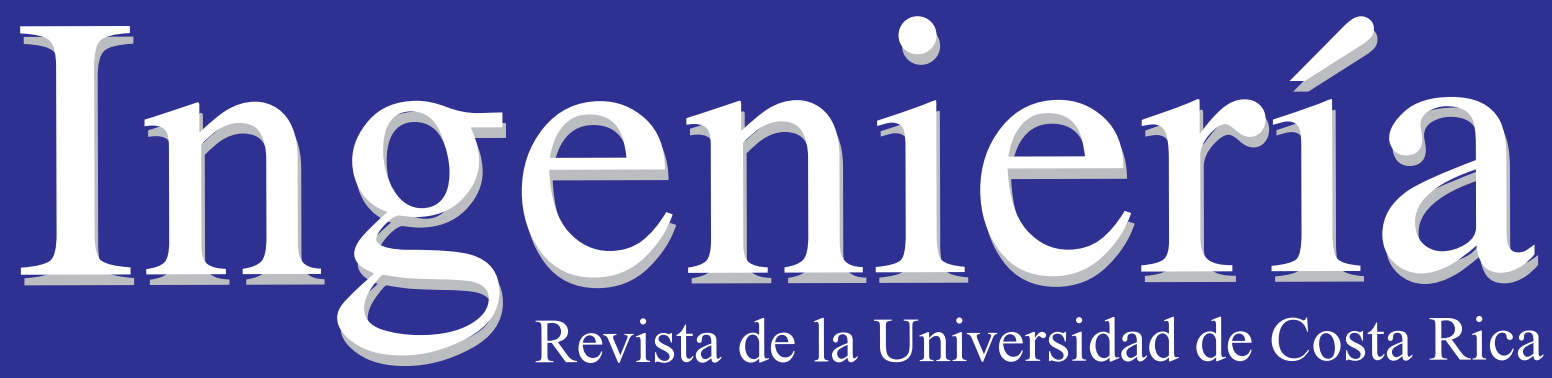

Revista de la Universidad de Costa Rica ENERO/ JULIO 2011 - VOLUMEN 21 Número (1)

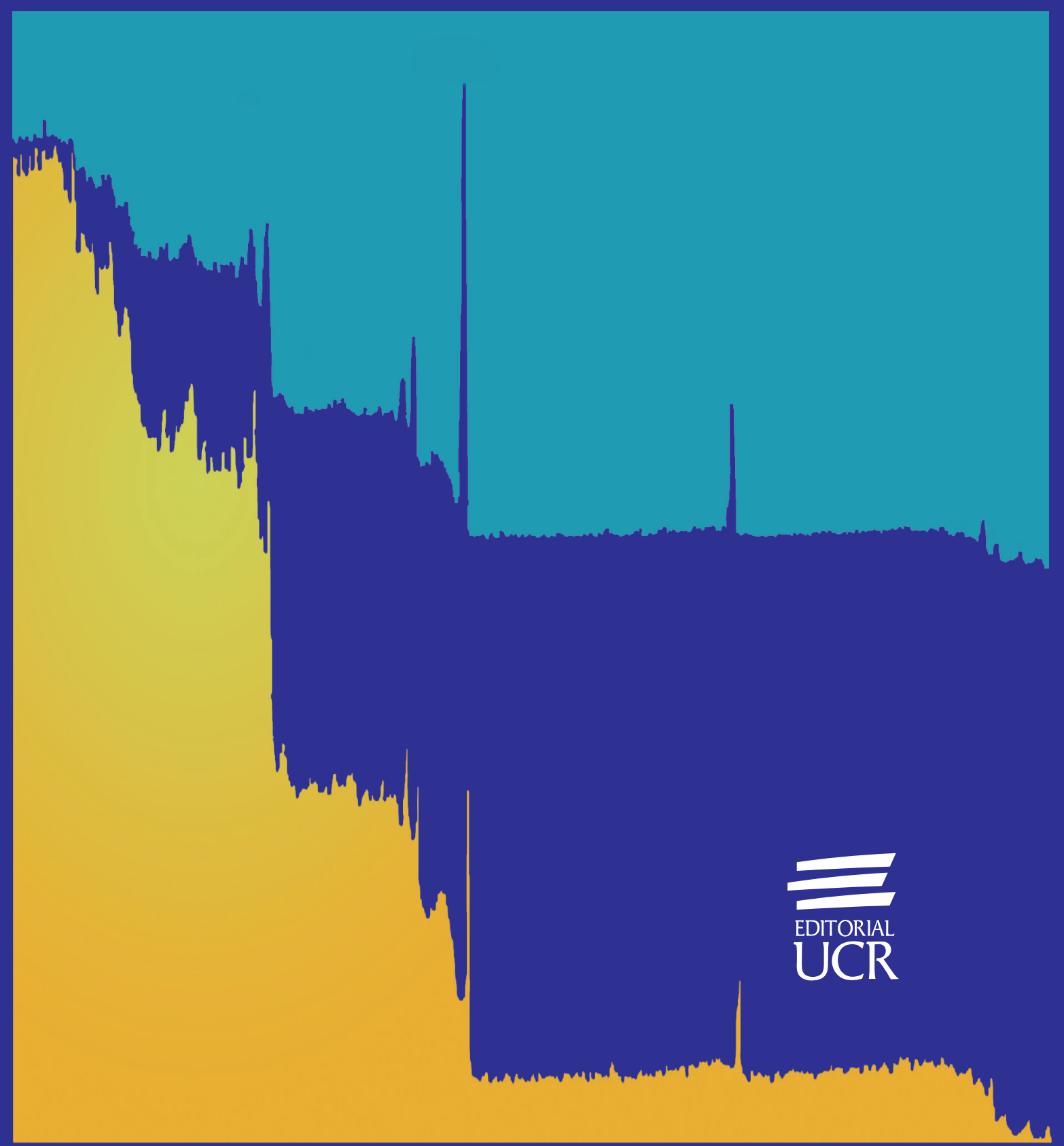




\title{
EL RUIDO: EFECTOS PSICOLÓGICOS Y SU INCIDENCIA ECONÓMICA
}

\section{Resumen}

\begin{abstract}
El ruido es un sonido que actúa como contaminante ambiental, tiene un efecto fisiológico y otro psicológico; en este trabajo se profundiza sobre el segundo, examinando las principales teorías y evidencias empíricas al día de hoy e igualmente se hace una consideración de cierto carácter económico. Se encuentran serias diferencias en los resultados de las investigaciones que pueden obedecer a la aplicación de protocolos y metodologías distintas como el de comparaciones en diferentes escenarios.
\end{abstract}

Palabras clave: Sonido, ruido, vibración, vibración mecánica, efecto fisiológico, efecto psicológico, contaminación ambiental.

\begin{abstract}
Noise is a sound that acts as environmental pollutant, and has a physiological and a psychological effects; this paper discusses the second, examining the major theories and empirical evidence to date and also includes slight economic consideration. They are serious differences in the results of investigations that may be due to the implementation of protocols and different methodologies such as the comparisons in different scenarios.
\end{abstract}

Keywords: Sound, noise, vibration, mechanical vibration, physiological effect, psychological effect, environmental pollution.

Recibido: 2 de setiembre del 2010 - Aprobado: 3 de marzo del 2011

\section{INTRODUCCIÓN}

La importancia que reviste el ruido como contaminante ambiental hace de este fenómeno una veta de investigación muy rica, dado los efectos que se derivan para la salud tanto de carácter fisiológico como psicológico.

La ingeniería juega un papel crucial en la búsqueda de la disminución en los niveles de ruido para armonizar la vida cotidiana y los espacios de trabajo, coadyuvando de esta manera a mejorar las condiciones de salubridad de los individuos que tanto lo requieren.

Este ensayo propone alertar sobre el grave riesgo que corren las personas que están sometidas a altos niveles de ruido y sus implicaciones en la salud, pero su énfasis se centra en los efectos psicológicos donde se hace una revisión teórica y se muestran algunas evidencias empíricas e igualmente se hace mención sobre su incidencia en la economía.

El ruido unido a otros fenómenos puede aumentar el grado de peligrosidad para la salud auditiva.

La interacción del ruido con otras exposiciones, tales como las vibraciones, agentes químicos o fármacos ototóxicos, puede aumentar el riesgo de hipoacusia (Instituto Nacional de Seguridad e Higiene en el Trabajo,2006 ).

El ruido lo definió la American Standard Association (ASA) en el año 1951 como "sonido no deseado" (Muscar, 2000). El ruido es un fenómeno acústico que tiene un componente físico y otro psicológico, el componente físico está relacionado con la intensidad sonora, frecuencia, naturaleza del tono e impulso y el tiempo de exposición; el psicológico está relacionado con la sensación que le produce 
a quien lo percibe, provocando de esta manera apreciaciones subjetivas.

Las fuentes del ruido son muy variadas y están relacionadas con las actividades de la vida cotidiana y la dinámica de la naturaleza.

Con respecto a las primeras, podemos en general distinguir las siguientes: Doméstica (aparatos y equipos de uso en el hogar, tonos de conversación y actividades); actividades industriales y de comercio (maquinaria, equipos, tipo de construcciones); transporte de carretera, ferroviario, aéreo, fluvial y marítimo (vehículos y equipos, trenes, aviones, barcos, maquinaría, pitos y sirenas); actividades agrícolas (maquinaria agrícola, molinos, desmotadoras, despulpadoras, trilladoras, equipos de transporte, ventiladores, motobombas, animales de labor); en construcción de infraestructuras y de edificios (maquinaria y equipos); guerras y ejercicios militares ( aviones, navíos, blindados, vehículos, proyectiles, bombas, voces entre otros); actividades religiosas, proselitismo político y manifestaciones de protesta (altavoces, pitos, ruido de personas); actividades de ocio y recreación entre otras (equipos de sonido, altavoces, música estridente, tonos de voz, movimientos corporales).

Los sonidos pueden generar sensaciones de agrado, rechazo o neutralidad y depende del tipo de población (edad, sexo, rasgo cultural, estado de salud, estado anímico y psicológico), origen, hora del día, actividad que realiza el receptor, grado de relación entre el receptor y el productor del ruido, grado de temor y el sentido de inevitabilidad que el ruido produce sobre un sujeto determinado y la situación temporal de la fuente de sonido.

El campo de la naturaleza destaca como fuentes de ruido el movimiento del viento, movimiento de las aguas de los ríos y océanos, la actividad sísmica, volcánica, truenos y ruido de animales.

El sonido es originado por vibraciones (movimientos oscilatorios de un cuerpo alrededor de un punto de referencia) que se propagan en todas las direcciones en un medio elástico, sólido, líquido o gaseoso, en forma de ondas longitudinales de presión acústica. Las ondas sonoras son generadas por un objeto en vibración que empuja las partículas de aire en contacto con el objeto y las transmite a las capas de aire adyacentes, dando como resultado un tren continuo de ondas de presión que se propaga alejándose desde el objeto en vibración.

Lo que el oído humano percibe como sonido no es otra cosa que variaciones en la presión acústica del aire provocadas por la energía irradiada de una fuente, la presión acústica depende de la distancia a la que se encuentre la fuente y de las característica y condiciones del ambiente o campo acústico en el que se propagan las ondas sonoras; por lo tanto, la presión acústica como medida (efecto) no es necesariamente la cantidad de ruido que produce un equipo o una máquina; el sonido por consiguiente es una forma de energía mecánica. Es importante resaltar que no existe una diferencia desde el punto de vista físico entre sonido y ruido (Procuraduría Ambiental y del Ordenamiento Territorial, 2006).

Las vibraciones mecánicas y el ruido como agentes que perturban el normal desenvolvimiento de la vida cotidiana han generado preocupación en el hombre desde la Antigüedad, Plinio el viejo (23-79 A.C.) narra en la "Historia Natural" que el ruido de las cataratas del río Nilo puede ser el responsable de la sordera en las gentes de aquella región (Rosen, 1974). Tanto en la antigua Grecia como en Roma, las actividades ruidosas fueron llevadas a sitios de menor concentración humana para evitar molestias entre los moradores de la ciudad.

La Epístola LVI de Seneca a Lucilio dice, “...figúrate todos los tipos de gritos que pueden repugnar a los oídos: cuando los atletas más fuertes hacen ejercicio y bracean con las manos cargadas de plomo, cuando se fatigan o hacen el fatigado siento los gemidos....por otro lado los ruidos intermitentes me molestan más que los continuos"(El ruido en la Antigua Roma, 2010).

Posteriormente, en Roma el emperador Julio Cesar prohibía hacer rodar los carros sobre las calles empedradas durante la noche y en algunas horas del día para evitar perturbar el descanso de las gentes de la ciudad. Durante el Medioevo, en Londres se prohibía castigar a las mujeres en la noche para no perturbar el sueño de los vecinos.

Bernardo Ramazini en el año de 1713 escribió un tratado alertando sobre los peligros para la salud auditiva provocados por los 
ruidos excesivos a lo largo del tiempo debido a los trabajadores caldereros que se dedicaban a martillar el cobre para conseguir diversas formas de las vasijas. Este autor explica que después de varios años los trabajadores caldereros terminan perdiendo la audición y que además la comunicación en estos lugares es imposible e igualmente que en la cabeza de los caldereros se forma un descontrol.

La mayor preocupación sobre los efectos del ruido cobra fuerza con la revolución industrial porque la dinámica de la misma introduce una mayor presencia del fenómeno mencionado, explicada esta situación por el aumento de la actividad económica, industrial y el crecimiento poblacional, son por lo tanto los centros industriales y la ciudad donde se comienza a sospechar seriamente lo nocivo que puede resultar el soportar altos niveles de ruido en un determinado tiempo. En el año de 1938 el Departamento de la Salud de Detroit clasificó 560 ocupaciones como ruidosas (Rosen, 1974).

Los estudios sobre los efectos del ruido en su mayoría se han centrado en las mediciones físicas tales como la intensidad y los daños auditivos que son los rasgos más visibles, descuidando en alguna medida los aspectos subjetivos y mentales que se pueden derivar de la exposición a esta fenomenología física.

\section{EVIDENCIAS EMPÍRICAS ACERCA DE LOS EFECTOS PSICOLÓGICOS DEL RUIDO}

Los resultados empíricos sobre los efectos del ruido son muy variados dada la complejidad de la evaluación del fenómeno como la metodología de evaluación implementada.

Uno de los trabajos pioneros sobre los efectos psicológicos del ruido se debe a Zepler y otros (1973), quien estudió el transporte como productor de ruido y vibración y el grado de respuesta humana cobijando las perturbaciones en el sueño y los efectos subjetivos sobre desempeño y confort.

A continuación se examinan algunos efectos psicológicos del ruido y explicaciones sobre sus diversos resultados.

\subsection{Efectos del ruido sobre el estrés y conducta violenta.}

El ruido como factor estresante ha sido investigado por Babisch y otros (2001), Babisch (2002), Babisch (2005), Griefahn y otros (2000), Jarup y otros (2005), Ouis (2002). Existe evidencias de una alta correlación entre el grado de valoración subjetiva y el malestar inducido por el ruido, la respuesta fisiológica humana por el malestar causado por el ruido del tráfico rodado resulta en un incremento del ritmo cardiaco como respuesta directa a un factor estresante y esto correlaciona bien (Raggam y otros, 2007).

Hay evidencias de admisiones crecientes en hospitales mentales en zonas de alto ruido y las relaciones entre exposición al ruido y depresión (Kryter, 1990; Ader, Cohen, 1993). El ruido también produce susto y efectos en el aumento del nivel de catecholamines, estas implican estrés y ansiedad (Ader, Cohen, 1993).

El estrés producido por el ruido ambiental está asociado a reacciones fisiológicas neuroendocrinas. Los niveles de hormonas del estrés en los fluidos del cuerpo, incluyendo adrenalina, noradrenalina y cortisol en orina, son indicadores útiles para valorar el estado agudo o crónico del estrés en las personas. Estas medidas son particularmente factibles en estudios epidemiológicos (Cohen, Kessler y Underwood, 1995; Babisch, Fromme y Beyer, 2001), es decir, la presencia de altas concentraciones en la orina de las substancias anteriormente enumeradas está asociada al estrés producido por ruido.

Los efectos del ruido sobre el rendimiento de las personas son distintos a los efectos de las molestias o los fisiológicos; existe escasa concordancia entre las medidas de estas diferentes respuestas, por ejemplo, una reacción violenta frente al ruido puede no ir acompañada de una pérdida de eficacia en el desarrollo de actividades, aunque puede producirse cambios en la eficacia y la persona considere el entorno aceptable, así también los cambios fisiológicos inducidos por ruido no se reflejan necesariamente en el rendimiento, sin embargo podría observarse una reducción en el rendimiento sin cambios fisiológicos detectables(Jones, Broadbent, 1995). 


\subsection{El ruido y los efectos en el sueño}

Existen varios estudios de laboratorio y de campo que demuestran que el ruido afecta la calidad del sueño y cuando el ruido es intermitente el problema es mayor que cuando es continuo, estos estudios datan desde los años setenta, ochenta y noventa del siglo pasado.

Los efectos del ruido en el sueño pueden ser inmediatos o posteriores a la exposición. Los efectos inmediatos son los que ocurren simultáneamente con el ruido o inmediatamente después de la emisión y los efectos posteriores o secundarios un día después al ruido o después de algunos días. Los efectos inmediatos pueden cuantificar el número y duración de veces que puede estar despierta la persona durante el periodo de sueño, etapas del sueño y ritmo (Pirrera, De Valck y Cluydts, 2010 ).

La posibilidad de despertarse por el ruido depende de la etapa del sueño, de los sonidos de fondo, el umbral del durmiente es particularmente alto en el sueño profundamente lento. Los efectos secundarios se pueden separar en informes de perturbaciones subjetivas del sueño y objetivas. La gente mayor se queja más del ruido nocturno que los jóvenes y puede deberse a que duran más horas despiertas o a que son más sensibles a dichos ruidos (Muzet, 2007).

Estudios sobre la incidencia del ruido producido por el tráfico rodado han encontrado que el ruido continuo tiene menos efecto, que el ruido intermitente, sobre la calidad del sueño y el humor; los movimientos del cuerpo después de cada ruido se aumentaron con el nivel del ruido intermitente (Ohrstrom y Rylanker, 1982; Thiessen, 1983). Posteriormente, se ha confirmado un mayor efecto nocivo del ruido intermitente frente al continuo en la calidad del sueño en un cuestionario, durante la mañana, cuando este excede los $60 \mathrm{~dB}(\mathrm{~A})$ (Ohrstrom y Rylanker, 1990).

Sin embargo, las conclusiones no son las mismas para otros estudios de laboratorio sobre la incidencia del ruido por tráfico y el sueño, encontrándose que no hay correlación (Eberhardt, Strate y Berlin,1987), también se halló que las perturbaciones en sueño son leves y se da al paso de niveles en las vías (Vernet, 1983). El poligrama monitoreado del sueño entre sujetos expuestos a nivel de ruido máximo en avión y trenes no reporto diferencias (Osada,Tsunanhima, Yoshida,Ogawa y Ohokubo, 1972).

Sobre una y variadas fuentes de ruido se observa tendencias similares en la calidad, molestias y alteraciones en el sueño; mientras que con respecto a los ruidos combinados se despertaban más temprano que los de un único tipo de ruido (Pyoung, Myung y Jin, 2010).

Un estudio realizado sobre ruido-reacción con una muestra de 300 no encontró relación significativa, mientras que con muestras por encima de 1000 fueron significativas (Job, 1996), estos resultados fueron anteriormente confirmados con medidas muy similares (Hede y Bullen,1982; Bullen, Hede y Job,1991;Job, Bullen y Burguess, 1991), igualmente se señala que cuando los datos son agrupados para áreas más pequeñas la correlación es alta.

Los hallazgos donde no se confirma correlación entre perturbaciones en el sueño ocasionadas por ruido puede deberse a muestras estadísticas pequeñas, como también el no tener en cuenta los niveles máximos de ruido y también puede incidir la actitud de las personas frente a la fuente de ruido que es un modificante de gran alcance a las respuestas del ruido ambiental, además, es necesario reconocer que la interpretación de los datos es difícil porque no existe un uso estándar en la medida del poligrama (Carter, 1996).

Cuando comienza a ver daños auditivos el grado de respuesta de un individuo frente al ruido no es el mismo, por lo tanto, no es comparable la actitud de un sordo o con cierto grado de sordera de quien no lo es, y en tal caso no hay adaptación al ruido sino que se está frente a una limitación orgánica, de ahí que toca examinar este hecho (Carter, 1996).

Aunque los efectos agudos sobre exposición al ruido se aceptan bien, las consecuencias a largo plazo sobre la salud se obstaculizan por resultados contradictorios y, aparte de los defectos metodológicos, la carencia de claridad conceptual en diseños de investigación se ha culpado de las inconsistencias pero se ha tratado raramente, por lo tanto, se debe abordar con seriedad este tema para examinar por que se presentan estos resultados (Lercher, 1996). 
Dado lo complejo del estudio de los sonidos medioambientales y su evaluación psicológica se recomienda tener presente varios puntos de vista: análisis de la física métrica, contramedidas para reducir el ruido no deseado, factores del entorno acústico, además, establecer estándares de calidad ambiental del ruido donde se tenga en cuenta antecedentes sociales que conlleven a evitar la producción de ruidos indeseados (Kuwano, 2008).

\subsection{Efectos del ruido en la vida cotidiana}

Se deben resaltar los efectos del ruido como obstáculo para las relaciones interpersonales. Un ambiente ruidoso puede causar aislamiento y frustración que pueden manifestarse en síntomas psicosomáticos o agresividad.

Se debe resaltar que es muy importante las condiciones ambientales para que se de un desarrollo óptimo de la persona.

\section{CONSIDERACIONES ECONÓMICAS}

La muerte temprana, enfermedades y ausencias laborales causan un importante coste económico a la sociedad. Estos factores parecen tener un proceso en ascenso debido a las nuevas condiciones ambientales, hábitos de las personas como a las relacionadas con la organización y nuevas exigencias laborales. La condiciones laborales aportan una importante participación dentro de estos costos y no es de menos que el ruido puede estar causando importantes ausencias laborales como factor estresante; además la no participación laboral por variadas causas puede estimular la pobreza, especialmente en la población económicamente activa.

Los problemas de salud mental, estrés, depresión, suicidio y condiciones relacionadas con el estrés son la causa más grande en estos momentos de muerte temprana a nivel de Europa; junto a formas de vida destructivas y las enfermedades psicosomáticas, causan un sufrimiento inmenso a la gente y sus familias, así como un gran costo económico en la sociedad (Levi, 2005).
El costo económico de los problemas de salud mental en los 15 estados miembros de la CEE (Comunidad Económica Europea) para el año 2000 está estimado entre 3 a $4 \%$ del PIB de estos países y para el año 2003 su cálculo está en promedio del $3.5 \%$ y su valor será de 325.000 millones de euros (Levi, 2005). Monto nada despreciable, lo que amerita tener en cuenta desde el punto de vista de salubridad, capital humano y costo económico.

Para el año 2004 según estimativos, la mortalidad por causa laboral en España supondría un coste medio en términos de pérdida de productividad de 800 millones de euros al año, y en torno a 150000 años potenciales de vida perdidos cada año, además se producirán cerca de 80000 nuevos casos de enfermedades de origen laboral (28 000 osteomusculares, 11 000 piel, 10000 sordera, 7500 alteraciones mentales, 5000 tumores malignos) (García, Gadea y López, 2007). Cabe además señalar un subregistro del $64 \%$ de casos incidentes de enfermedades profesionales (García, Gadea y López, 2007).

A modo de ejemplo para ilustrar la magnitud del ruido, se muestra en la tabla siguiente la evolución de la percepción del ruido en el trabajo para los sectores de actividad en la economía española .

Se observa que a pesar de los esfuerzos realizados a nivel de la empresa y por parte del gobierno español, vigilando y auspiciando la mejora en la seguridad e higiene en el trabajo, se encuentra que más del $20 \%$ de los trabajadores del sector industrial están expuestos a ruido elevado y muy elevado. Teniendo en cuenta los datos del año 2007, los trabajadores afectados por este tipo de ruido serian aproximadamente 800000 en la industria, 600000 en la construcción, 79350 en el sector agrario y 648 000 en los servicios.

\section{CONCLUSIONES}

- Debido a los problemas fisiológicos y psicológicos que pueden derivarse de las exposiciones a ruidos, debe haber un compromiso decidido de combate al ruido innecesario que debe partir de hacer una gran 
pedagogía para que las legislaciones y las normativas al respecto sean acatadas.

- Las investigaciones sobre las fuentes y efectos del ruido deben ocupar un lugar importante al día de hoy, cubriendo diversas áreas del conocimiento, como la ingeniería, arquitectura, medicina, psicología, psiquiatría, sociología, biología, geografía, economía y derecho entre otras,

- $\quad$ Se debe tener en cuenta las dimensiones que viene ocupando el ruido como agente de contaminación ambiental a través de sus diversas fuentes y las consecuencias que se derivan.

- Es necesario tener en cuenta el tipo de normativa y metodología a seguir para medir los efectos del ruido tanto a largo como a corto plazo.

Tabla 1. Exposición de trabajadores al ruido elevado y muy elevado en el puesto de trabajo por sectores de actividad en términos porcentuales en España

Encuesta

No

III

IV

V

VI 2007

\begin{abstract}
Año
\end{abstract}
Agrario

Industrial

$17.7 \%$

$22.3 \%$

$21 \%$

$24.8 \%$
Sectores de Actividad

$\begin{array}{ccc}\text { Construcción } & \text { Servicios } & \text { Total } \\ 8 \% & 4.8 \% & 8.4 \% \\ 12 \% & 5.4 \% & 10.3 \% \\ & & \\ & & \\ 20.4 \% & 5 \% & 9.3 \% \\ & & \\ 21.9 \% & 4.8 \% & 10.5 \%\end{array}$

Fuente: Elaboración propia a partir de la III, IV, V y VI Encuesta Nacional de Condiciones de Trabajo del Ministerio de Trabajo y Asuntos Sociales y el Instituto Nacional de Seguridad e Higiene en el Trabajo de España.

\section{NOTAS}

1. Este ensayo se basa en el trabajo de grado para obtener el título de Diploma de Estudios Avanzados (DEA) de Doctorado en Ingeniería Mecánica y Fabricación en la Universidad Politécnica de Madrid, nutriéndose con nuevas revisiones bibliográficas.

2. Las encuestas anteriores a la sexta, el formulario de preguntas se contestaba en la empresa y era el mismo para empresario y trabajador; en cambio, la sexta obedece al formulario del trabajador y su contestación estuvo fuera del puesto de trabajo y en el mayor anonimato para evitar sesgos.

\section{BIBLIOGRAFÍA.}

Ader, R.; Cohen, N. (1993). "Psychoneuroimmunology: conditioning and stress", Annual. Rev. Psychol. 44, 53-85.

Babisch, W.(2005). "Noise and health". Environmental Health Perspectives. 113(1), 14- 15.

Babisch, W.(2005). "The noise/stress concept, risk assessment and research needs". Noise \& Health. 4 (16), 1-11.

Babisch, W; From me, H; Beyer, A; Ising, H.(2001). "Increased catecholamine levels in urine in subjects exposed to road traffic noise". Environment International. 26, 475-481. 
Bullen, R.B; Hede, A. J.; Job, R.F.S. (1991). "Community reaction to noise from an artillery range”. Noise Control Eng. J. 37, 115-128.

Carter, N.L.(1996). "Transportation noise, sleep, and possible after-effects". Environment International. 22(1), 105-116.

Cohen, S.; Kessler, R.C.; Underwood, G. L.(1995). "Strategies for measuring stress in studies of psychiatric and physical disorders". In: Cohen, S.; Kessler, R.C.; Underwod, G. L., editors. A guide for health and social scientists.(pp. 3-26.) New York: Oxford Univ. Press,

Eberhardt, J.L; Strate, L.O; Berlin, M.H. (1987) "The influence of continuous and intermittent traffic noise sleep". Journal of sound and Vibration. 116, 445-464.

El ruido en la Antigua Roma: Un epigrama de Marcial y una epístola de Séneca.(2010) creado: 25/08/ 2010. http://www.ruidos.org/ Documentos/Epigrama_Marcial.html

García, A. M.; Gadea, R.; López, V.(2007). Impacto de las enfermedades laborales en España. Madrid: Instituto Sindical de Trabajo, Ambiente y Salud (ISTAS).

Griefahn, B.; Schuemer-Kohrs, A.; Schuemer, R.; Moehler, U.; Mehnert. (2000) "Physiological, subjective, and behavioral responses during sleep to noise from rail and road traffic". Noise \& Health. 3(9),59-71.

Hede, A. J; Bullen, R. B. (1982) Aircraft noise in Australia a survey of community reaction. National Acoustic Laboratories Report No 88. Canberra ACT: Australian Govermment Publishig Service.

Instituto Nacional de Seguridad e Higiene en el Trabajo.(2006) Guía técnica para la evaluación y prevención de los riesgos relacionados con la exposición de los trabajadores al ruido. Ministerio de trabajo e Inmigración de España. Revisado en: http://www.insht.es/InshtWeb/contenidos/ normativa/guíastécnicas/Ficheros/guía técnicaruido.pdf

Jarup,L.; Dudley, M.; Babisch, W; Houthuijs, D.; Pershagen, G. et al. (2005) "Hypertension and exposure to noise near airports (HYENA): Study desing and noise exposure assessment". Environmental Health Perspectives. 113,1473-1478.

Job, R.F.S. (1996) "The influence of subjective reactions to noise on health effects of the noise”. Environment International, 22 (1), 93-104.

Job, R.F.S.; Bullen, R.B.; Burgess, D.H.(1991). "Noise-induced reaction in a work community adjacent to aircraft runways: the Royal Australian Airforce”. In: Lawrence, A, (Eds). Proceedings of Inter-Noise '91: the cost of noise.(Vol 2, pp.985998). Poughkeepsie, NY: Noise Control Foundation.

Jones, D. M.; Broadbent, D. E.(1995). "Rendimiento humano y ruido". En: Manual de Medidas Acústicas y Control del Ruido.(3 ed., pp. 24.1-24.26) España: Mc Graw Hill.

Kryter, K.D.(1990) "Aircraft noise and social factors in psychiatric hospital admission rates: a reexamination of some data". Psychol. Med. 20, 395-411.

Kuwano, S.(2008). "Evaluación psicológica del entorno acústico a lo largo del tiempo”. Revista de Acústica. 38 ( 3- 4), 17-35.

Lercher, P.(1996)."Environmental noise and health: An integrated research perspective". Environment International. 22(1), 117-129.

Levi, L. (2005) "Working Life and Mental Health- A Challenge to Psychiatry?". WPA Section Report (pp. 53-57). Stockholm: WPA Section on Ocupational Psychiatric Karoliska Institute.

Muscar, E.(2000) "El ruido nos mata". En Anales de Geografía (Vol 20, pp 149-161) Madrid: Universidad Complutense de Madrid.

Muzet, A. (2007). "Environmental noise, sleep and health". Sleep Medicine Reviews.11, 135-142.

Ohrstrom, E.; Rylanker, R.(1982). "Sleep disturbance by road traffic noise- a aboratory study on after effects". Journal of Sound and Vibration. 84, 87-103.

Ohrstrom, E.; Rylanker, R.(1990). "Sleep disturbance by road traffic noise- a laboratory study on number of noise events". Journal of Sound and Vibration. 143, 93-101.

Osada, Y.; Tsunanhima, S.; Yoshida, K.; Ogawa, S.; Ohokubo, C.(1972). "Effects of train and jet aircraft noise on sleep". Bull. Inst. Publ. 
Health. 21, 133-138.

Ouis, D.(2002) “Annoyance caused by exposure to road traffic noise: an update". Noise \& Health. 4 (15), 69-79.

Pirrera, S.; De Valck, E.; Cluydts, R. (2010). "Nocturnal road traffic noise: A review on its assessment and consequences on sleep and health". Environment International. 36, 492-498.

Procuraduría Ambiental y del Ordenamiento Territorial.(2006) "Contaminación por ruido y vibraciones: Implicaciones en la salud y calidad de vida de la población urbana". México, D.F: Autor.

Pyoung,J.L.; Myung,H.S.;Jin,Y.J.(2010).“Effectf different noise combinations on sleep, as assessed by general questionnaire". Applied Acoustics. 71, .870-875.

Raggam, R. B.; Cik, M.; Holdrich, R. R.; Fallast, K.; Gallasch, E.; Fend, M.; Lackner, A.; Marth, E.(2007) "Personal noise ranking of road traffic: Subjective estimation versus physiological parameters under laboratory conditions". International Journal of Hygiene and Environmental Health. 210, 97-105.

Rosen, G.(1974) "A backward glance at noise pollution". American Journal of Public Health. 64 (5), 514- 517.

Thiessen, G.(1983). "Effect of intermittent and continuous traffic noise on various sleep characteristics and their adaptation". In: Rossi G. (Eds). Proceedings fourth international congress on noise as a public health problem. (Vol 2, pp. 995-1005) Milano, Italy: Centre Richerche E studi Amplifon.

Vernet, M.(1983).“Comparison between train noise and road noise annoyance during sleep". Journal Sound and Vibration. 87, 331-335.

Zepler, E.E; Sullivan, B.M; Rice, C.G; Griffin, M.J; Oldman, M; Dickinson, P.J... Large, J.B.(1973) "Human response to transportation noise and vibration". Journal of Sound and Vibration, 28 (3), 375-401.

\section{SOBRE EL AUTOR}

\section{Jaime Gómez}

Ingeniero Mecánico, Universidad Antonio Nariño, Bogotá Colombia. Economista, Universidad Autónoma de Colombia, Bogotá Colombia. Magíster en Economía, Pontifica Universidad Javeriana, Bogotá Colombia. DEA en Economía, Universidad Autónoma de Madrid, España. DEA en Ing. Mecánica, Universidad Politécnica de Madrid, Madrid España. Especialista en Diseño Mecánico por Computador, Universidad Antonio Nariño, Bogotá Colombia. Candidato a Doctor en Economía, Universidad Autónoma de Madrid. Candidato a Doctor en Ingeniería Mecánica, Universidad Politécnica de Madrid. Profesor Universitario y Consultor. Correo electrónico: jaimea.gomez@estudiante. uam.es-jaimegomezwalteros@hotmail.com. 